\title{
ENTENDIMIENTO DEL CONCEPTO MOL LOGRADO POR LOS ESTUDIANTES EN UN CURSO DE QUÍMICA GENERAL DE CORTE CONSTRUCTIVISTA
}

\author{
Carlos Utria Echeverría*, Roberto Figueroa Molina ${ }^{* *}$
}

\begin{abstract}
This work intends to discuss the conceptual understanding of a general chernistry course (chemistry 3001), with a constructivist approach. A group of 28 students participated in an analysis of a series of repeated measurement obtained through the elaboration of conceptual maps about the concept of mole they handle within three intances: a) before studying the isue; b) inmediatilly after studying it; and c) after studying other related concepts, 8 of those students were interviewed to make then escribe their conceptual maps. The analysis of the repeated measures indicated that significatives differences existed arnong all the three instances. This proved that a course with a constructivist approach significantly contributed to promote the development of the conceptual understanding of the mole concept among the students. Some of the recommendations consisted in developing and implementing a consistent teaching methods that promote a stronger understandíng of scientific concepts among studens, and to accomplish a better training of teachers of natural science to make them teach toward a better conceptual understanding among others.
\end{abstract}

Key words: Conceptual Understanding, General Chemistry, Mole, Constructivist and Conceptual Maps.

\section{RESUMEN}

El propósito de esta investigación fue estudiar el entendimiento conceptual logrado en un curso de Química General ("Quím 3001"), con enfoque constructivista. Un grupo de 28 estudiantes participó en un estudio de medidas repetidas obtenidas, a través de mapas conceptuales acerca del concepto mol elaborados en tres tiempos durante el curso: 1) antes de estudiar el concepto; 2) inmediatamente luego de que se estudiara; y 3) luego de estudiar otros conceptos relacionados. El análisis de medidas repetidas, indicó que existían diferencias significativas entre todos los tres tiempos, especialmente los primeros dos. Se evidenció, por tanto, que el curso de Química General con enfoque constructivista, contribuyó significativamente a promover el desarrollo del entendimiento del concepto mol en los estudiantes. Algunas recomendaciones ofrecidas fueron: desarrollar e implantar métodos de enseñanza que promuevan el entendimiento de conceptos científicos, capacitar a los profesores de ciencias naturales con el propósito de que enfoquen su instrucción al entendimiento de los conceptos, entre otras.

Palabras clave: entendimiento conceptual, química general, mol, constructivismo ymapas conceptuales.

\footnotetext{
*Profesor Universidad del Atlántico. C_utria@hotmail.com

*** Profesor Universidad del Atlántico. roberfigue@hotmail.com 


\section{Introducción}

Según la Asociación Americana para el Avance de la Ciencia (AAAS por sus siglas en inglés), una premisa fundamental para los cambios en la sala de clases, es que las escuelas no necesitan encargarse de enseñar más y más contenido, sino focalizar lo esencial de la literatura científica y enseñar esto más efectivamente (Wandersee, Mintzes y Novak, 1994).

La enseñanza de las ciencias requiere metas claras y modelos no tradicionales, que utilicen los procesos de inquirir y de exploración, fundamentados en la curiosidad natural de los seres humanos, para promover el desarrollo de destrezas asociadas a la cultura científica (Rivas, 1999). Mediante estas estrategias de aprendizaje activo, se pretende promover el entendimiento de conceptos básicos en el estudiante, como requisito indispensable para que pueda comprender las teorías fundamentales de las ciencias. Entendimiento conceptual o de conceptos, se define como la habilidad que tienen los estudiantes para reconocer, interpretar, explicar e ilustrar adecuadamente las conexiones e interconexiones entre los conceptos subordinados de un macroconcepto y entre éstos con otros conceptos relacionados (Aguirre, 1998).

En química, por ejemplo, la enseñanza de los cursos medulares debe fomentar en el estudiante la capacidad para diseñar sus propios experimentos científicos (Arce, 1997). Esa capacidad, requiere un entendimiento de orden superior de los conceptos que componen las teorías aceptadas. En consecuencia, cualquier proceso instruccional en ciencias, que falle en el intento de lograr el entendimiento de conceptos debería considerarse inefectivo.

Existe un considerable número de investigaciones que han ilustrado los problemas que tienen los estudiantes en adquirir el entendimiento de los conceptos y principios químicos (Novick \& Nussbaun, 1981; Yarroch, 1985; Nurrenbern \& Pickering, 1987; Sawrey, 1990; Pickering, 1990; Peterson \& Treagust, 1989). Los problemas que destaca esta literatura son: (1) el aprendizaje de los estudiantes es predominante por repetición o memorización, más bien que por construcción activa de su conocimiento; (2) los estudiantes no conocen Los atributos más importantes de los conceptos, ni pueden establecer las relaciones entre conceptos necesarios, para entender un macroconcepto o idea de química; (3) la instrucción puede fallar al no ofrecer suficientes experiencias en las cuales el que aprende pueda reflexionar y expresar la forma en que está estableciendo las relaciones.

Existe mucha controversia acerca de qué fomenta el entendimiento conceptual en una disciplina. En química, varios investigadores han examinado las relaciones entre la solución de problemas numéricos y el entendimiento conceptual. Algunos investigadores, indican que el entendimiento conceptual de los estudiantes es inadecuado para solucionar problemas químicos que requieren transferencia (Gabel, 1993; Gabel, Sherwood y Enochs, 1991; Anamuah-Mensah, 1986 y Bunce, Gabel y Samuel, 1991), es decir, problemas químicos donde el estudiante tiene que utilizar y relacionar conceptos para poder solucionarlos. De igual modo, los estudios demostraron que los estudiantes frecuentemente solucionan los problemas numéricos usando un acercamiento algorítmico exclusivamente.

Otros estudios (Cros, Chastrette \& Fayol, 1988; Rowell, Dawson 8 Lindon, 1990), han demostrado que la instrucción tradicional en química modifica las concepciones de los estudiantes, pero no en gran medida. Algunas veces, la instrucción crea errores en las 
concepciones científicas o conceptos erróneos ("misconceptions"). Por ello, se ha identificado la necesidad de explorar estrategias instrucciónales alternas, para fomentar el entendimiento conceptual profundo.

El estudio de estas innovaciones se hace cada día más necesario en la educación, para tener evidencia del valor educativo de estos esfuerzos, y si conviene continuar con ellos. Uno de los cursos transformados mediante estos esfuerzos es el curso de Química General enseñado por la Dra. Josefina Arce, en la Universidad de Puerto Rico, Recinto de Río Piedras, el cual sirve de base a este estudio.

La pregunta que orientó la investigación fue la siguiente: ¿Logra el curso de Química General de corte constructivista promover un significativo aumento en el entendimiento del concepto mol en los estudiantes?

\section{Método}

En este estudio se utilizó un diseño de medidas repetidas. Se realizó un análisis de tipo cuantitativo a las puntuaciones obtenidas a través de mapas conceptuales construidos por todos los estudiantes $(n=28)$.

\section{Participantes}

Los estudiantes que tomaron el curso con la Dra. Arce en el 1er. semestre del año académico, fueron los participantes del estudio. Estos eran 20 mujeres y 8 hombres, con una edad promedio de 19.2 años y un promedio de ingreso a la universidad de 3.43 .

\section{Recopilación de datos}

El mapa de conceptos, es un procedimiento basado en la teoría de Ausubel (Novak, 1984). Esta técnica se ha utilizado en diferentes estudios para evaluar el entendimiento conceptual en Química (Pendley, Bretz y Novak, 1994 y Nakhleh, 1994). Los mapas de conceptos consisten en jerarquizar y relacionar conceptos a través de proposiciones. Se utilizan para que el estudiante exprese el entendimiento que tiene de un concepto, a través de sus propias construcciones y estilos de aprendizaje. Los mapas de conceptos son técnicas adecuadas que posibilitan otorgar puntuaciones a los estudiantes de acuerdo a lo que ejecuten en ellos.

Para propósitos de esta investigación, los mapas conceptuales fueron construidos en tres tiempos. Para recopilar la información, se le pidió a los 28 estudiantes que construyeran un mapa conceptual del concepto mol. Los estudiantes tuvieron 45 minutos para elaborar el mapa conceptual, en cada uno de los tres tiempos.

En el tiempo 1, al comenzar el semestre (agosto, 2000), se le pidió a cada uno de los estudiantes que elaboraran un mapa conceptual del concepto mal, para explorar el conocimiento previo que poseían los estudiantes acerca de este concepto antes de comenzar el estudio del mismo. En el tiempo 2, se les entregó a los estudiantes el mapa conceptual que cada uno de ellos elaboró en el tiempo 1, para que corrigieran, agregaran, eliminaran o lo reelaboraran, partiendo de lo que estimaran conveniente. En el tiempo 2, los mapas conceptuales se realizaron luego de que se enseñó el concepto mol en la sala de clases (octubre, 2000). En el tiempo 3, poco antes de finalizar el semestre (noviembre, 2000), se les entregaron nuevamente, los mapas conceptuales creados en los dos tiempos anteriores para que los corrigieran, agregaran conceptos, eliminaran conceptos o 
reelaboraran los mismos y, para que establecieran nuevas relaciones con otros conceptos estudiados.

\section{Análisis de datos}

El análisis cuantitativo, se realizó mediante el desarrollo y uso de una rúbrica (Utría, 2000), para otorgar valor a la ejecución en los mapas conceptuales. La puntuación está distribuida de acuerdo con las características del mapa, tales como: jerarquía, proposiciones, ramificaciones, entrecruzamiento y ejemplos. La base fundamental del esquema de puntuación es la teoría cognoscitiva del aprendizaje (Ausubel, 1968).

Las puntuaciones de todos los mapas conceptuales de los estudiantes, fueron otorgadas por uno de los investigadores utilizando la rúbrica. En adición, una estudiante doctoral de educación con experiencia en la técnica de mapas de conceptos participó como un segundo evaluador de todos los mapas, en los tres tiempos, utilizando la misma rúbrica. La puntuación final de cada uno de los mapas en los tres tiempos fue acumulativa.

Los dos jueces evaluaron, independientemente, todos los mapas en los tres tiempos. Se determinó la confiabilidad entre los dos jueces que evaluaron los mapas por medio de la prueba estadística de Spearman's rho. Los resultados demostraron coeficientes de confiabilidad altos entre los dos jueces, en las tres medidas (Spearman $r=0.908,0.989$ y 0.995, respectivamente). En el caso que las puntuaciones discreparan, los dos jueces se pusieron de acuerdo con respecto a la puntuación de estos mapas; las puntuaciones logradas en consenso fueron las utilizadas en el análisis.

Las puntuaciones obtenidas de las ejecuciones mostradas en los mapas conceptuales, por parte de los estudiantes en los tres tiempos, se analizaron estadísticamente, calculando la media y la desviación estándar para cada administración. Para evaluar diferencias en las tres medidas repetidas del grupo de intervención, se utilizó la prueba estadística MANOVA (SPSS).

\section{Resultados}

El cuestionamiento que guió este estudio, enfocaba el aumento en el entendimiento conceptual logrado a través del curso. La inspección de los promedios de las medidas repetidas, obtenidas a través de los mapas, sugiere que hubo un gran aumento entre las puntuaciones del tiempo 1 y tiempo 2, y que los mismos continuaron aumentando, aunque en menor magnitud, en el tiempo 3 (Véase tabla 1). La prueba MANOVA para medidas repetidas arrojó que existían diferencias estadísticamente significativas entre los tres tiempos de los mapas conceptuales $(F=57.24$, $g l=2, p=0.000)$.

Tabla 1. Promedio y desviación estándar de las medidas repetidas.

\begin{tabular}{|c|c|c|}
\hline Etapas & Promedio & $\begin{array}{c}\text { Desviación } \\
\text { Estándar }\end{array}$ \\
\hline Tiempo 1 & 1.536 & 1.934 \\
Tiempo 2 & 12.250 & 7.511 \\
Tiempo 3 & 15.536 & 8.500 \\
\hline
\end{tabular}


De igual modo, se identificaron diferencias entre el tiempo 1, y el tiempo 3 (F 80.09, gl= 1.27, p 0.000), y entre el tiempo 2 y, el promedio del tiempo 1 y el tiempo 3 de los mapas conceptuales $(F=14.16, g l=1,27, p=0.001)$.

De estos resultados, se desprende que los estudiantes mostraron un aumento progresivo, y estadísticamente significativo, a través de los distintos tiempos de medición, lo que indica que su entendimiento del concepto mol fue aumentando considerablemente, según transcurría su proceso de aprendizaje en el curso de corte constructivista.

\section{Discusión}

Se examinaron los cambios logrados en el entendimiento del concepto mol, mostrado por el grupo de 28 estudiantes, observándose un incremento progresivo del entendimiento del concepto a través del tiempo. De este hallazgo se desprende que el curso de Química General "Quim 3001, que enseña la Dra. Josefina Arce, mediante un enfoque constructivista fomenta el desarrollo del entendimiento de concepto mol en los estudiantes.

Los cambios en el entendimiento del concepto mol que se reflejaron en las medidas repetidas realizadas a los mapas conceptuales elaborados por los estudiantes podría interpretarse como que esta innovación que se está realizando en la facultad de ciencias naturales, es útil para el proceso de aprendizaje de los conceptos que se cubren en el curso de Química General. Las estrategias de enseñanza implantadas podrían estar añadiendo eficiencia al curso de Química General (Quim 3001") para desarrollar en los estudiantes el entendimiento de conceptos.

El entendimiento de conceptos en Química es importante para que los estudiantes comprendan los procesos científicos inherentes a esta asignatura de modo significativo. Lo anterior abre la posibilidad de que si se difunde su práctica en la facultad de Ciencias Naturales, podría mejorar la instrucción en comparación con la enseñanza tradicional. Esto es consistente con estudios que enfatizan en la implantación de estrategias con enfoques constructivistas para el desarrollo del entendimiento de conceptos (Nakhleh, 1992). De esta forma, se facilita que los educadores puedan ayudar a los estudiantes a entender y discernir las condiciones bajo las cuales cada concepto puede ser internalizado significativamente en su esquema conceptual.

De igual modo, el desarrollo del entendimiento del concepto mal lograda por el estudiantado del curso de Química General de la sección enseñada mediante estrategias constructivistas es consistente con autores que señalan que una buena instrucción en Química debe modificar las percepciones que tienen los estudiantes de los conceptos. Es decir, deben facilitar un buen entendimiento conceptual (Cros, Chastrette \& Fayol, 1988; Rowell, Dawson \& Lindon, 1990). Al examinar las puntuaciones obtenidas en los mapas fundamentados en el concepto mol se notó un aumento progresivo.

En la revisión de literatura se encontró, además, que se necesitan modelos instruccionales viables y efectivos para el entendimiento de conceptos en Química (Freedman, 1997; Nakhleh, 1994). Los hallazgos de este estudio, sugieren que las innovaciones y los modelos de enseñanza que se utilizan en el curso de Química General estudiado, son efectivos para ese propósito. Dentro de las estrategias de enseñanza y aprendizaje se encuentran las tareas en grupos de aprendizaje cooperativo en y fuera del salón de clase. Se utilizan, además, demostraciones interactivas, basadas en el proceso de predicción —observación- explicación y basadas en la realización de experimentos 
en el salón de clases. El profesar dirige a los estudiantes, a través de un proceso donde todos predicen lo que puede ocurrir frente a una situación dada y luego lo investigan, para clarificar sus ideas. Más aún, en el laboratorio se expone a los estudiantes a experimentos, en los que tienen que desarrollar sus propios diseños experimentales para obtener datos o resolver un problema. Por lo anterior, los educadores en Química, pueden encontrar en estos modelos de enseñanza recursos metodológicos disponibles para su quehacer educativo.

A la luz de lo antes presentado, se puede llegar a las siguientes conclusiones: (1) El enfoque constructivista fue aparentemente efectivo para promover el entendimiento del concepto mol en un curso de Química General. (2) El entendimiento del concepto mol aumentó progresivamente en los estudiantes que tomaron el curso bajo estudio, evidenciada mediante el análisis del entendimiento antes de estudiar el concepto en clase, al terminar de discutirlo y luego de un tiempo de discutido.

Las conclusiones antes presentadas hacen pertinentes las siguientes recomendaciones: (1) Los departamentos de ciencias deben desarrollar e implantar métodos de enseñanza con enfoque constructivista para promover el entendimiento de conceptos científicos. (2) Los profesores universitarios del área de ciencias deben capacitarse para implantar procesos de enseñanza y aprendizaje que propendan por mejorar el nivel de entendimiento conceptual de los estudiantes. (3) Debe prestarse especial atención al desarrollo e implantación de estrategias efectivas para ayudar a los estudiantes a sustituir conceptos erróneos o incompletos. (4) Se deben evaluar otros cursos donde se estén modificando los métodos de enseñanza, para conocer el valor educativo de estos esfuerzos.

\section{Agradecimientos}

Los autores agradecen a la doctora Milagros Bravo, a la doctora María Medina y a la doctora María Aguirre por sus acertadas intervenciones. Las recomendaciones de cada una de ellas, hicieron que esta experiencia fuera para nosotros un crecimiento incesante, como profesionales y como personas.

Reconozco, además, la aportación a esta investigación de Elena Maldonado, quien desinteresadamente nos brindó su ayuda como evaluadora de los mapas conceptuales.

Finalmente, queremos agradecer, de forma muy especial, a la doctora Josefina Arce y a los estudiantes del curso de química general, por permitirnos realizar esta investigación y llevar a feliz término este trabajo.

\section{Bibliografía}

Aguirre, M. (1989) Open-ended questions: An alternative mode to assess the students' performance in concepto development and use of scientific vocabulaiy. Unpublished dissertation, State University of New York at Buffalo, p. 12.

American Association for the Advancement of Science., (2001) Science for All Americans: Project. Washington, D.Cp. pp.19-22. 
Anamuah - Mensah, J., (1986) Cognitive Strategies Used by chemistry students to solve volumetric analysis problems. Journal of Research in Science Teaching, 23 [9], 1986, pp. 759-769.

Arce, J., (1997) Student-designed experiments in scientific lab instruction. Journal of College Teaching, 1997, pp. 114-118.

Ausubel, D., (1968) Educational Psychology: A cognitive view. New York: Holt. RINEHART, \& WINSTON, Bunce, D., Gabel, D., \& Samuel, K., (1991) Enchancing chemistry problern-solving achievment using problern categorization. Journal of Research in Science Teaching, 28 [6], pp. 505-521.

Bunce, D.; Gabel, D. \& Samuel 1<. Enchancing chemistry problem-solving achievment using problem categorizafien. Journal of Research in Science Teaching, 28 [6], 1991, pp. 505-521.

Cros, D.; Chastrette, M. \& Fayol, M. (1988) Conceptions of second year university students of some fundamental notions of chemistry. International Journal of Science Education, 10 [3], 1988, pp. 331- 336.

Freedrnan, M. (1997) Relationship among laboratory instruction, attitude toward science, and achievement in science knowledge. Journal of Research in Science Teaching, 34, [4], 1997, pp. 343-357.

Gabel, (1993) D. Use of the particle nature of matter in developing conceptual understanding. Journal of Chemical Education, 70, [3], 1993, pp. 193-194.

Gabel, D.; Sherwood, R. \& Enochs, L. (1991) Problem-solving skills of high school chemistry students. Journal of Research in Science Teaching, 21, [2], 1991, pp. 221 233.

Nakhleh, M. (1994) How can research uncover what students are learning? Journal of Chemical Education, 71 [3], 1994, pp. 201-203.

Nakhleh, M. (1992.) Why some students don't learn chemistry: Chemical misconceptions. Journal of Chemical Educations, 69 [3], 1992, pp. 191-196.

Novak, J. (1994) Application of advances in learning theory and philosophy of science to the improvement of chemistry teaching. Journal of Chemical Education, 61 [7], 1984, pp. 607-612.

Novick, S. \& Nussbaum, J. (1978) Junior high school pupils understanding of the particulate nature of matter: An interview Study. Science Education, 62 [3], 1978, pp. 273-281.

Nurrenbern, S. \& Pickering, M. (1987). Concept learning versus problem solving: is there a difference? Journal of Chemical Education, 64 [6], 1987, pp. 508-510.

Pendley, B.; Bretz, R. \& Novak, J. (1994) Concept maps as a tool ft assess learning in chemistry. Journal of Research in Science Teaching, 71 [1], 1994, pp. 9-15. 
Peterson, R. \& Treagust, D.; Grade (1989). 12 students misconceptions of covalent bonding and structure. Journal of Chemical Education, 66 [6], 1989, pp. 459-460.

Pickering, M. (1990) Further studies un concept learning versus problem solving. Journal of Chemical Education, 67 [3], 1990, pp. 254-255.

Rivas, A. (1999). La importancia de la naturaleza de las ciencias en el desarrollo de una cultura científica. El Gran Educador, 1 [2], 1999, pp. 7-8.

Rowell, J.; Dawson, C. \& Lyndon, H. (1990). Changing misconceptions: A challenge to science educators. International Journal Science Education, 12 [2], 1990, pp. 167-175.

Sawrey, B. (1990).Concept learning versus problem solving: Revisited. Journal of Chemical Education, 67 [3], 1990, pp. 253-254.

Utría, C. (2000) Entendimiento conceptual logrado por estudiantes en un curso de química general de corte constructivista. Tesis de grado. Universidad de Puerto Rico, 2000.

Wandersee, J.; Mintzes, J. \& Novak, J. (1994) Research on alternative conceptions in science. In D. Gabel (Ed.), Handbook of research on science teaching and learning New York: Macmillan, 1994, pp. 177-210.

Yarroch, W. (1985) Student understanding of chemical equation balancing. Journal of Research in Science Teaching, 22 [5], 1985, pp. 499-459. 